\title{
Involvement of connexin 43 in the acute hyperosmotic stimulus-induced synthesis and release of vasopressin in the supraoptic nucleus of rats
}

\author{
SHAN JIANG ${ }^{1 *}$, YONG-QIANG WANG ${ }^{2 *}$, CHENG-FENG XU $^{1}$, YA-NA LI $^{1}$, RONG GUO $^{1}$ and LING LI $^{1}$ \\ ${ }^{1}$ Department of Physical Medicine and Rehabilitation, The First Affiliated Hospital, Chinese PLA General Hospital, \\ Beijing 100048; ${ }^{2}$ Department of Opthalmology, Jinan Military General Hospital, Jinan, Shandong 250031, P.R. China
}

Received September 12, 2013; Accepted May 2, 2014

DOI: $10.3892 / \mathrm{mmr} .2014 .2400$

\begin{abstract}
There is evidence that astroglial connexin43 $(\mathrm{Cx} 43)$ in the supraoptic nucleus (SON) is required for the hyperosmolarity-induced increase in Fos protein expression in magnocellular neurosecretory cells (MNCs). In the present study, the role of astroglial Cx43 in the synthesis and release of vasopressin (VP) by MNCs in the SON subjected to hyperosmotic stimulus was examined. The results revealed that the VP levels in the SON and plasma were increased following acute hyperosmotic stimulus. Treatment of MNCs with Cx43-specific antisense oligodeoxynucleotides (ASODN), which temporarily reduced $\mathrm{Cx} 43$ protein production, limited the VP synthesis and release induced by a hyperosmotic stimulus. Similarly, the addition of gap junction and Cx43 hemichannel blockers also attenuated the VP synthesis and release induced by an acute hyperosmotic stimulus. A high extracellular $\left[\mathrm{Ca}^{2+}\right]\left(\left[\mathrm{Ca}^{2+}\right]_{0}\right)$ has been demonstrated to reduce the gap junction activity or opening probability of Cx54 hemichannels. Notably, it was identified that high $\left[\mathrm{Ca}^{2+}\right]_{0}$ attenuated the VP synthesis and release induced by acute hyperosmotic stimulus, while low $\left[\mathrm{Ca}^{2+}\right]_{0}$ had a weak or no effect. These results suggested that Cx43 participates in the VP synthesis and release induced by hyperosmotic stimulation in the SON.
\end{abstract}

\section{Introduction}

The maintenance of stable osmolarity in the extracellular fluid is essential for physiological functioning and is partly achieved via the regulation of vasopressin (VP) release from

Correspondence to: Professor Ling Li, The First Affiliated Hospital, Chinese PLA General Hospital, 51 Fucheng Road, Beijing 100048, P.R. China

E-mail: landjiang@126.com

${ }^{*}$ Contributed equally

Key words: hyperosmotic stimulus, vasopressin, connexin43, magnocellular neurosecretory cells, supraoptic nucleus the neurohypophysial axon terminals of the magnocellular neurosecretory cells (MNCs) in the supraoptic nucleus (SON) and the paraventricular nucleus (PVN) (1). Several studies demonstrated that astrocytes in the SON and PVN also have important roles in regulating osmotic pressure (2). Under hypotonic pressure, taurine, an amino acid that is abundant in astrocytes (3), is released upon astrocyte swelling and activates the glycine receptors on MNCs in the SON and PVN to inhibit the activation of MNCs and VP release (2). By contrast, under hyperosmotic pressure, MNCs are activated and release VP with the passive decreased release of taurine by astrocytes (2).

Accumulating evidence has further revealed the positive role of astrocytes under hyperosmotic conditions. Previous studies demonstrated that an acute hypertonic stimulus (HS) upregulated the expression of c-fos, glial fibrillary acidic protein (GFAP) and connexin43 (Cx43) in SON astrocytes $(4,5)$. In addition, inhibiting the activity of astrocytes with fluorocitrate (FCA) prevented the response of the MNCs to HS and attenuated the release of VP, indicating a positive role of astrocytes in regulating hypertonicity. It is suggested that astrocytes in the SON may receive osmotic information from the peripheral osmoreceptors and then subsequently regulate MNCs (6). However, the mechanism by which this regulation occurs has not been fully elucidated. Previously, it was demonstrated that carbenoxolone (CBX), a gap junction blocker, interrupted the hyperosmotic stimulation-induced increase in Fos protein expression in MNCs (7), suggesting that connexins, the biogenetic precursors of the gap junction, which are mainly expressed in astrocytes in the SON, are required for the subsequent activation of MNCs, therefore supporting the hypothesis that Cx43 may be involved in the regulation of VP synthesis and release under acute hyperosmotic stimulation.

Accordingly, the present study was designed to determine whether astroglial $\mathrm{Cx} 43$ in the SON may have a role in VP synthesis and release by MNCs following acute hyperosmotic stimulation. Cx43-specific antisense oligodeoxynucleotides (ASODN) were employed to temporarily reduce $\mathrm{Cx} 43$ protein levels (8), and the effect on acute hyperosmotic stimulus-mediated VP release was assessed. In addition, the effect of gap junction blockers, Cx43 hemichannel blockers (CBX or Gap26) and high extracellular $\left[\mathrm{Ca}^{2+}\right]\left(\left[\mathrm{Ca}^{2+}\right]_{0}\right)$ on the acute hyperosmotic stimulus-induced VP release in the SON 
and plasma was assessed. The study suggested that $\mathrm{Cx} 43$ may have an important role in the regulation of VP synthesis and release under hypertonic pressure in astrocytes.

\section{Materials and methods}

Animal models. Adult male Sprague-Dawley (SD) rats, weighing 200-250 g, were provided by the Animal Center of the Chinese PLA General Hospital (Beijing, China). The experimental procedures were conducted in accordance with the Guidelines for the Use of Animals in Neuroscience Research (published in the Membership Directory of the Society, pp 27-28, 1992) (9) and were also approved by The Committee of Animal Use for Research and Education of the Chinese PLA General Hospital. The rats were housed under environmentally controlled conditions $\left(22^{\circ} \mathrm{C}\right.$, a 12 -h light/dark cycle with light from 6:00 to 18:00) with ad libitum access to a standard laboratory diet and water. Prior to HS, the rats were allowed to adapt to their new environment for one week.

A total of 90 rats were randomly divided into nine groups: Normal control $(\mathrm{n}=10)$; isotonic control $(\mathrm{n}=10)$; hypertonic stimulus (HS; $1.5 \mathrm{M}, \mathrm{n}=10$ ); Cx43-specific ASODN+HS $(\mathrm{n}=10)$; CBX+HS ( $\mathrm{n}=10)$; Gap26+HS $(\mathrm{n}=10) ; 0 \mathrm{mM} \mathrm{Ca}^{2+}+\mathrm{HS}$ $(\mathrm{n}=10) ; 1 \mathrm{mM} \mathrm{Ca}^{2+}+\mathrm{HS}(\mathrm{n}=10)$ and $2.5 \mathrm{mM} \mathrm{Ca}^{2+}+\mathrm{HS}(\mathrm{n}=10)$.

In order to examine the role of $\mathrm{Cx} 43$ in the hyperosmotic stimulus-induced VP synthesis and release, a sterile polyethylene tube $(0.5 \mathrm{~mm}$ in diameter) was inserted into the lateral ventricle under anesthesia, as described previously (7). The animals were allowed to recover from the surgery for five days. In the CBX+HS and Gap26+HS groups, the gap junction blocker CBX (Sigma, St. Louis, MO, USA; $50 \mu \mathrm{g} / \mathrm{rat}$, $10 \mu \mathrm{g} / \mu \mathrm{l})(32,33)$ or Gap26 (VCYDKSFPISHVR; Cx43 hemichannel blocking peptide; Severn Biotech, Ltd., Kidderminster, Worcestershire, UK; $2 \mu \mathrm{l} / \mathrm{rat}, 300 \mu \mathrm{mol} / \mathrm{l})$ was delivered into the lateral ventricle via the pre-embedded tube. Cx43-specific antisense oligonucleotides were generated based on the sequence 5'-ACTCCAGTCACCCAT-3', which is specific for $\mathrm{Cx} 43$, as confirmed by sequence alignment using the National Center for Biotechnology Information GenBank (https://www. ncbi.nlm.nih.gov/genbank/) and has been demonstrated to be effective in knocking down $\mathrm{Cx} 43$ expression (34-36). Cx43-specific ASODN (synthesis by Shanghai Sangon Biotech Co., Ltd., Shanghai, China; $2 \mu \mathrm{l} / \mathrm{rat}, 1.5 \mathrm{mmol} / \mathrm{l}$ ) was also delivered into the lateral ventricle $(35,36)$. In the $0 \mathrm{mM}$ $\mathrm{Ca}^{2+}+\mathrm{HS}, 1 \mathrm{mM} \mathrm{Ca}^{2+}+\mathrm{HS}$ and $2.5 \mathrm{mM} \mathrm{Ca}^{2+}+\mathrm{HS}$ groups, the rats were pre-injected with artificial cerebrospinal fluid (CSF) containing 0,1 and $2.5 \mathrm{mM} \mathrm{Ca}^{2+}$, respectively, into the lateral ventricle via the pre-embedded tube.

A total of $2 \mathrm{~h}$ following the delivery of all of the reagents, hyperosmotic stimulation was performed. For hyperosmotic stimulation, a $\mathrm{HS}(5,7)(1.5 \mathrm{M} \mathrm{NaCl}$ solution, $5.5 \mathrm{ml} / \mathrm{kg}$ body weight) was injected into the caudal veins. For the isotonic control, isotonic solution (IS; $0.15 \mathrm{M} \mathrm{NaCl}$ solution, $5.5 \mathrm{ml} / \mathrm{kg}$ body weight) was used in the same way as described previously $(5,7)$. The normal control group did not receive any of the above treatments. All of the animals were carefully monitored during the injection period, and no signs of pain or discomfort were noted. Following the injection, the rats were housed under the same conditions as before and the animals were sacrificed at $45 \mathrm{~min}$ post-injection.
In order to exclude the effect of the reagents themselves on VP synthesis and release, another 60 rats were divided into six groups: $\mathrm{Cx} 43$-specific ASODN ( $\mathrm{n}=10)$; $\mathrm{CBX}(\mathrm{n}=10)$; Gap26 $(\mathrm{n}=10) ; 0 \mathrm{mM} \mathrm{Ca}^{2+}(\mathrm{n}=10) ; 1 \mathrm{mM} \mathrm{Ca}^{2+}(\mathrm{n}=10)$ and $2.5 \mathrm{mM} \mathrm{Ca}^{2+}$ $(\mathrm{n}=10)$. These rats were not administered the HS.

Plasma VP content radioimmunoassay. Plasma VP concentrations were measured using a radioimmunoassay kit. Blood samples $(0.5 \mathrm{ml} / 100 \mathrm{~g}$ body weight $)$ were collected from the femoral veins at baseline immediately prior to IS or hypertonic solution injection ( 0 min time-point) or $90 \mathrm{~min}$ following injection of IS or hypertonic solution, and centrifuged within $1 \mathrm{~h}$ of sampling at $1,500 \mathrm{x}$ g for $15 \mathrm{~min}$ at $4^{\circ} \mathrm{C}$. The plasma samples were separated and stored as aliquots in plastic tubes at $-70^{\circ} \mathrm{C}$ until use. All of the samples were examined using the vasopressin ${ }^{125}$ I RIA kit (DiaSorin Company, Stillwater, MN, USA), according to the manufacturer's instructions. The assays were performed blindly by a member of laboratory staff.

Immunofluorescencemicroscopy andmorphometric analysis. Prior to sacrifice, the rats were administered a supplemental dose of sodium pentobarbital (60 mg/kg, i.p.) and perfused transcardially with $100 \mathrm{ml}$ cold phosphate buffer (PB; $0.1 \mathrm{M}$, $\mathrm{pH}$ 7.4), followed by $500 \mathrm{ml} 4 \%$ paraformaldehyde in $0.1 \mathrm{M}$ $\mathrm{PB}$. The brains were removed immediately following perfusion and placed in $20 \%$ sucrose (W/V) in $0.1 \mathrm{M} \mathrm{PB}$ overnight at $48^{\circ} \mathrm{C}$. Each forebrain, including the hypothalamus, was cut at $30 \mu \mathrm{m}$ thickness on a cryostat (Cryostal; Leitz, Wetzlar, Germany). The forebrain slices through the SON of the hypothalamlus were prepared for immunofluorescence staining, as described previously (32). Briefly, the sections were rinsed in $0.1 \mathrm{M}$ phosphate-buffered saline (PBS) containing $0.03 \%$ Triton X-100 for $30 \mathrm{~min}$ at room temperature. Following another wash with $0.1 \mathrm{M}$ PBS, the sections were incubated for $48 \mathrm{~h}$ at $48^{\circ} \mathrm{C}$ with rabbit against VP polyclonal antiserum (1:1,000; Chemicon, Billerica, MA, USA; AB1565). A set of sections were incubated in normal immunoglobulin (Ig) $\mathrm{G}$ and used as negative controls. Following incubations with the primary antibody, the sections were washed for $1 \mathrm{~h}$ in $0.1 \mathrm{M}$ PBS and then incubated for $24 \mathrm{~h}$ at $48^{\circ} \mathrm{C}$ with the secondary antibodies (fluorescein isothiocyanate-conjugated goat anti-rabbit IgG; 1:500; Sigma). Following a final rinse in $0.1 \mathrm{M}$ PBS, the sections were mounted on glass slides. No positive signal was observed on the negative control sections.

Morphometric analysis was performed as described previously $(6,7)$. A single optical slice $(2.3 \mu \mathrm{m})$ image was obtained from each SON section with a confocal microscope (FluoView 300; Olympus, Tokyo, Japan) and processed further with Adobe Photoshop (8.0; Adobe Systems, San Jose, CA, USA). Semi-quantitative mean fluorescence intensities (MFI) were calculated using Image Pro Plus software (7.0; Media Cybernetics, Siver Spring, MD, USA). The immunofluorescent cells were counted in the whole SON area in each of the six serial sections in a set, from which an average number of labeled cells per section was derived. In examining the VP protein levels, the fluorescence intensity was normalized to a standard curve $(0-255)$ to allow for comparison (and averaging) across individual experiments. The average background fluorescence emission was deter- 

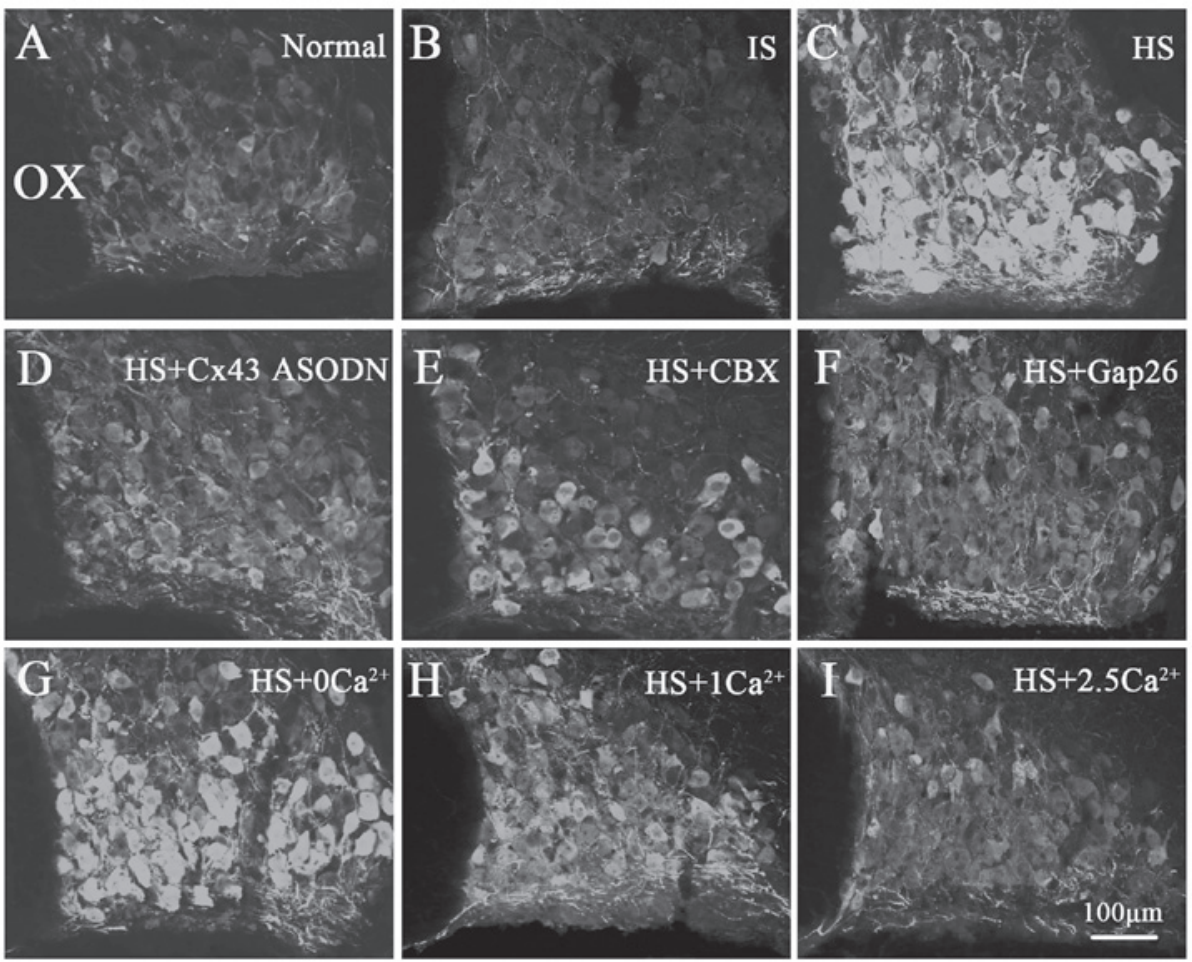

Figure 1. (A) VP expression in SON neurons 45 min following IS or HS treatment of normal control, (B) IS control, (C) HS, (D) HS+Cx43 ASODN, (E) HS+CBX, (F) $\mathrm{HS}+\mathrm{Gap} 26,(\mathrm{G}) \mathrm{HS}+0 \mathrm{Ca}^{2+}$, (H) $\mathrm{HS}+1 \mathrm{Ca}^{2+}$ and (I) $\mathrm{HS}+2.5 \mathrm{Ca}^{2+}$ rats. Scale bar, $100 \mu \mathrm{m}$. Magnification, x100. VP, vasopressin; SON, supraoptic nucleus; IS, isotonic solution; HS, hypertonic stimulus; $\mathrm{Cx} 43$, connexin43; ASODN, antisense oligodeoxynucleotides.

mined and subtracted from the values obtained for positively labeled cells.

Semi-quantitative polymerase chain reaction (PCR) analysis. Total RNA was obtained from the SON using TRIzol (Invitrogen Life Technologies, Carlsbad, CA, USA) reagent according to the manufacturer's instructions. cDNA was generated from $1 \mu \mathrm{g}$ of total RNA by using the PrimeScript RT-PCR kit (Takara Bio Inc., Shiga, Japan). Each PCR was conducted in a $20 \mu 1$ volume with $100 \mathrm{pmol}$ of each $5^{\prime}$ and $3^{\prime}$ gene-specific primer and $1 \mu 1$ of diluted cDNA. PCR cycle conditions were 35 cycles of $94^{\circ} \mathrm{C}$ for $1 \mathrm{~min}, 62^{\circ} \mathrm{C}$ for $1 \mathrm{~min}$ and $72^{\circ} \mathrm{C}$ for $1 \mathrm{~min}$, followed by a final extension at $72^{\circ} \mathrm{C}$ for $10 \mathrm{~min}$. The levels of $\beta$-actin were used as a control for the sample amount. The PCR products were resolved by staining with ethidium bromide and were run by electrophoresis in $1.2 \%$ agarose gels. The results were visualized under ultraviolet illumination using a Gel Doc apparatus (Gene Genius Bioimaging system; Syngene, Cambridge, UK). Images of the PCR bands were analyzed semi-quantitatively using Image $\mathbf{J}$ analysis software (1.45e; National Institutes of Health, Bethesda MD, USA). The sequences of the primers (Invitrogen, Shanghai, China) used were as follows: VP, $374 \mathrm{bp}$, forward 5'-CGG CAA AGG GCG CTG CTT CG-3' and reverse 5'-CCG GGG CTT GGC AGA ATC CA-3'); $\beta$-actin, $540 \mathrm{bp}$, forward 5'-GTG TTC CGC TCT AGG CAC CAA-3' and reverse 5'-CTC TTT GAT GTC ACG CAC GAT TT-3'.

Western blot analysis. The protein extracts were obtained from the SON. Briefly, the samples for western blot analysis were removed from the $-80^{\circ} \mathrm{C}$ freezer and immediately homog- enized at $4^{\circ} \mathrm{C}$ with $0.5 \mathrm{ml}$ lysis buffer $[0.01 \mathrm{M}$ Tris- $\mathrm{HCl}$ buffer (pH 7.6) containing $0.25 \mathrm{M}$ sucrose, $0.1 \mathrm{M} \mathrm{NaCl}, 1 \mathrm{mM}$ EDTA and $1 \mathrm{mM}$ phenylmethylsulfonylfluoride]. The supernatant was collected following centrifugation at $10,000 \mathrm{x} \mathrm{g}$ for $10 \mathrm{~min}$. The protein concentration in the supernatant was determined by a Bradford assay. The aliquots of the clarified homogenized liquid, containing $30 \mu \mathrm{g}$ of protein, were denatured in a sample buffer [0.1 M Tris- $\mathrm{HCl}$ buffer ( $\mathrm{pH}$ 6.8) containing $0.2 \mathrm{M}$ DTT, 4\% SDS, $20 \%$ glycerol and $0.1 \%$ bromophenol blue]. The samples were then analyzed by $10 \%$ SDS-PAGE and transferred to polyvinylidene fluoride membranes (Bio-Rad, Hercules, CA, USA). The primary antibodies included rabbit anti-Cx43 polyclonal antibody (Sigma; 1:1,200) and rabbit polyclonal anti-GAPDH (Sigma; 1:1,000). The secondary antibody was horseradish peroxidase conjugated goat anti-rabbit IgG (Sigma; 1:500). The protein levels in the Cx43-ASODN group were expressed as the percentage of control values.

Statistical analysis. All data are expressed as the mean \pm standard error of the mean. Between-group comparisons were made using one-way analysis of variance with Dunnett's post-hoc test or Dunnett's T3 test for multiple comparisons. Data were analyzed with SPSS (version 18.0; SPSS, Inc., Chicago, IL, USA). $\mathrm{P}<0.05$ was considered to indicate a statistically significant difference.

\section{Results}

Acute HS increases VP levels in the SON and plasma. Changes in the expression of VP in the SON following acute hyperosmotic stimulation were examined by immunostaining. 
A

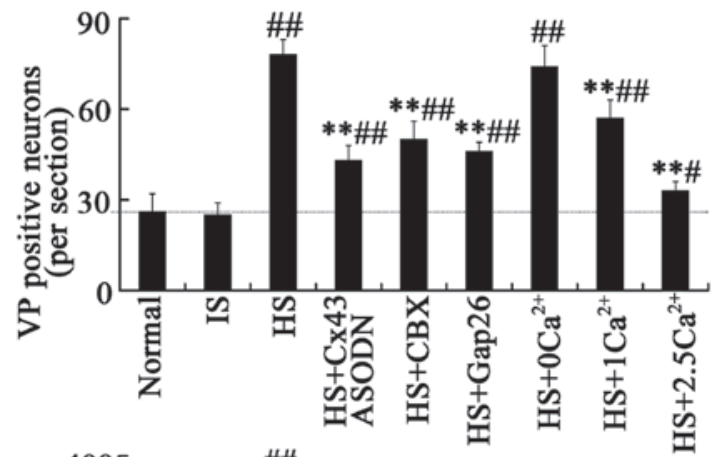

B

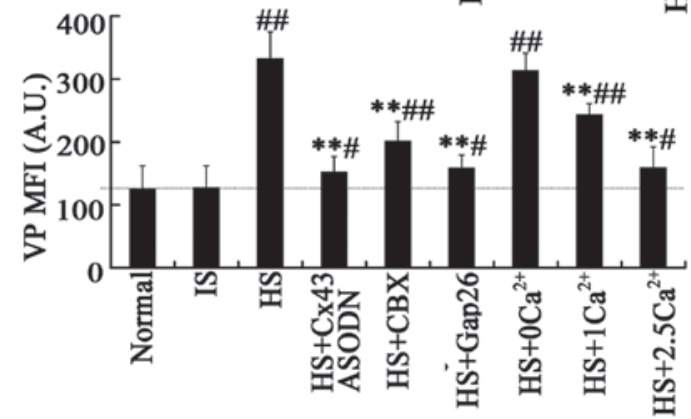

Figure 2. Comparison of the number of (A) VP-positive neurons and (B) MFI of VP-positive neurons $45 \mathrm{~min}$ following IS or HS treatment in the various treatment groups. ${ }^{*} \mathrm{P}<0.05,{ }^{* *} \mathrm{P}<0.01$ vs. the HS group; ${ }^{\#} \mathrm{P}<0.05,{ }^{\# \#} \mathrm{P}<0.01$, vs the normal group. VP, vasopressin; MFI, mean fluorescence intensities; IS, isotonic solution; HS, hypertonic stimulus; Cx43, connexin43; ASODN, antisense oligodeoxynucleotides.

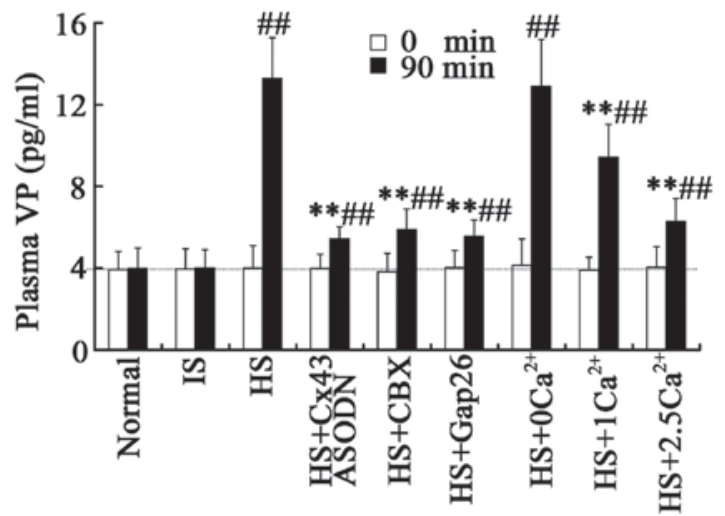

Figure 3. Changes in plasma VP concentrations $(\mathrm{pg} / \mathrm{ml})$ in the various treatment groups in response to HS. Open bars represent baseline plasma VP concentrations at $0 \mathrm{~min}$ (prior to IS or HS treatment). Solid bars represent plasma VP concentrations 90 min following IS or HS treatment. ${ }^{*} \mathrm{P}<0.05$, ${ }^{* *} \mathrm{P}<0.01$ vs. the HS group $(90 \mathrm{~min}) ;{ }^{\#} \mathrm{P}<0.05,{ }^{\# \#} \mathrm{P}<0.01$ vs. the normal group (0 min). VP, vasopressin; IS, isotonic solution; HS, hypertonic stimulus; Cx43, connexin43; ASODN, antisense oligodeoxynucleotides.

VP immunoreactivity was present in cells in the SON as demonstrated previously $(6,7)$. In the IS control group, few VP-positive SON neurons were observed, indicating low levels of VP (Fig. 1A and B). The mean number of VP-positive neurons per section were $25 \pm 4$ and $26 \pm 6$ in the IS control and normal control groups, respectively (Fig. 2A and B). Following acute HS treatment, VP immunostaining was enhanced (Fig. 1C) and the mean number of VP-positive neurons was significantly increased to $78 \pm 5$ neurons per section (Fig. 2A). The MFI of SON cells labeled with anti-VP was significantly higher in the HS group (332 \pm 43 A.U.; Fig. 2B)
$\mathbf{A}$
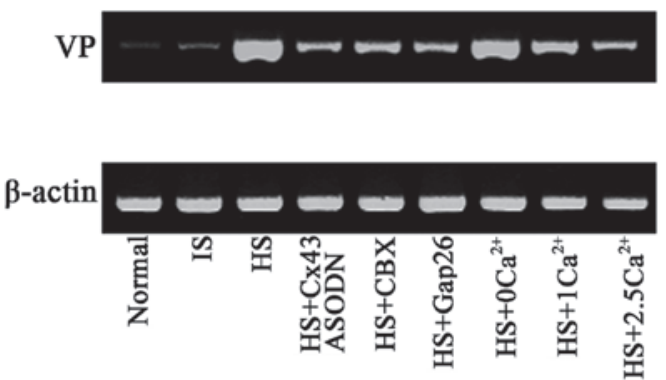

B

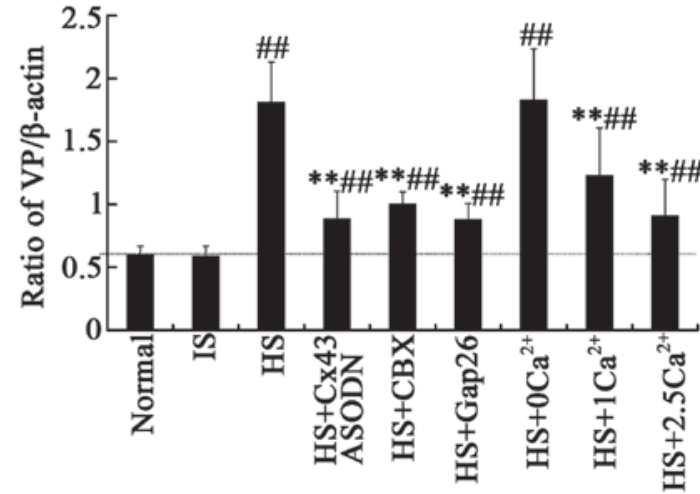

Figure 4. (A) Semi-quantitative polymerase chain reaction analysis demonstrating the mRNA expression of VP in SON at 45 min following HS treatment. $\beta$-actin was used as an internal control. (B) The ratio of VP/ $\beta$-actin in SON at 45 min following HS or IS treatment in each group. ${ }^{*} \mathrm{P}<0.05$, ${ }^{* *} \mathrm{P}<0.01$ vs. the HS group; ${ }^{*} \mathrm{P}<0.05,{ }^{\# \#} \mathrm{P}<0.01$, vs. the normal group. VP, vasopressin; IS, isotonic solution; HS, hypertonic stimulus; SON, supraoptic nucleus; Cx43, connexin43; ASODN, antisense oligodeoxynucleotides.

than that in the controls (normal control, 125 \pm 37 A.U.; IS control, 127 \pm 35 A.U.; Fig. 1A and B; Fig. 2B; P<0.01). In addition, VP mRNA expression was increased to $1.811 \pm 0.32$ in the HS group, compared with $0.598 \pm 0.07$ and $0.587 \pm 0.08$ in the normal control and IS control groups $(\mathrm{P}<0.01$, Fig. 4$)$, therefore suggesting that acute hyperosmotic stimulation in the SON stimulates VP synthesis.

Additionally, the effect of acute hyperosmotic stimulation on plasma VP levels was assessed. The baseline plasma VP levels in all of the groups were $3.95 \mathrm{pg} / \mathrm{ml}$. VP levels were not altered with IS treatment; however, acute HS treatment induced a three-fold increase in VP levels to $13.28 \pm 2 \mathrm{pg} / \mathrm{ml}(\mathrm{P}<0.01$, Fig. 3). These results further demonstrated that acute HS increased the synthesis and release of VP.

For the rats who did not receive HS treatment following the delivery of the reagents, the VP levels in the SON and plasma were not significantly different from those in the IS and normal groups (data not shown).

Cx43-specific ASODN attenuates the acute hyperosmotic stimulation-induced VP upregulation in the SON and plasma. ASODN is a short chain nucleotide that blocks the expression of a target gene by binding to and preventing the translation of mRNA, therefore temporarily reducing target protein production (10). Application of Cx43-specific ASODN results in a temporary knockdown of $\mathrm{Cx} 43$ protein and a decrease in the formation of new Cx43 gap junctions (8) (Fig. 5).

In the present study, Cx43-specific ASODN was used to detect the effect of $\mathrm{Cx} 43$ on the acute HS-induced VP upregulation in the SON and plasma. It was identified that 
A

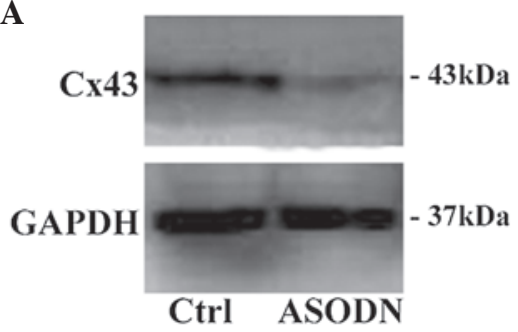

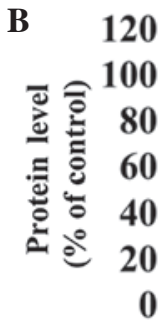

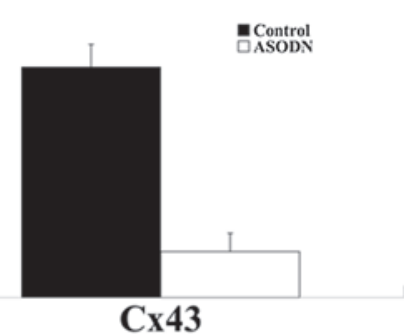

Figure 5. (A) Western-Blot analysis demonstrated tge protein expression of Cx43 in SON following Cx43-ASODN treatment. GAPDH was used as an internal control. (B) Cx43 levels (\% control) were markedly decreased following Cx43-ASODN treatment. ${ }^{* *} \mathrm{P}<0.01$ vs. control. Cx43, connexin43; ASODN, antisense oligodeoxynucleotides; SON, supraoptic nucleus; Ctrl, control.

Cx43-specific ASODN significantly decreased the acute HS-induced increase in VP levels and the expression in the SON and plasma. In the ASODN+HS group, the mean number of VP-positive neurons was lower (43 \pm 5 VP-positive cells per section, Figs. 1D and 2A) than that in the HS group $(\mathrm{P}<0.01)$, but remained higher when compared with that of the IS or normal control groups. An attenuation in the MFI (152 \pm 25 A.U.; Fig. 2B) of VP-positive neurons was also observed. Furthermore, VP mRNA expression was significantly decreased $(0.884 \pm 0.22$; Fig. 4$)$ in the $\mathrm{Cx} 43$-specific ASODN+HS group compared with that in the HS group, suggesting a decrease in VP synthesis in the SON.

Similarly, the VP concentration in the plasma was lowered $(5.42 \pm 1.2 \mathrm{pg} / \mathrm{ml})$ in the $\mathrm{Cx} 43$-specific ASODN-treated rats following HS. $(\mathrm{P}<0.01$; Fig. 3$)$, suggesting that $\mathrm{Cx} 43$ is required for the HS-mediated induction of VP.

$C B X$ and Gap26 attenuate the acute hyperosmotic stimulus-induced VP upregulation in SON and plasma. Cx43 hemichannels are the biogenetic precursors of gap junctions, which are the main connexons expressed in astrocytes (11-13). As aforementioned, the present study demonstrated that the expression of $\mathrm{Cx} 43$ was required for the HS-mediated induction of VP. To investigate the role of gap junctions or $\mathrm{Cx} 43$ hemichannels on the acute HS-induced synthesis and release of VP in MNCs, the rats were pre-treated with CBX, a gap junction blocker, or Gap26, a Cx43 hemichannel blocking peptide. Both CBX and Gap26 significantly attenuated the acute HS-induced increase in VP levels in the SON and plasma. In the $\mathrm{HS}+\mathrm{CBX}$ group, a $30 \%$ reduction in the mean number $(50 \pm 6)$ and a $40 \%$ reduction in MFI (201 \pm 31 A.U.) of VP-positive neurons were observed compared with the HS group ( $\mathrm{P}<0.01$; Fig. 1E, Fig. 2). Similar results were observed in the HS+Gap26 group (number of VP-positive neurons, 46 \pm 3 ; MFI, 158 21 A.U.; Fig. 1F, Fig. 2). Furthermore, semi-quantitative PCR analysis demonstrated that VP synthesis induced by HS was also inhibited by $\mathrm{CBX}$ and Gap26. The ratio of $\mathrm{VP} / \beta$-actin was reduced to $1 \pm 0.1$ and $0.877 \pm 0.13$ in the HS+CBX and HS+Gap26 groups, respectively (Fig. 4).

It was also identified that both CBX and Gap26 pre-treatment prevented the HS-induced increase in plasma VP levels. The VP concentrations were $5.88 \pm 1$ and $5.55 \pm 0.8 \mathrm{pg} / \mathrm{ml}$ in the $\mathrm{HS}+\mathrm{CBX}$ and HS+Gap26 groups, respectively ( $\mathrm{P}<0.01$; Fig. 3).

In addition, glycyrrhizic acid, which is structurally similar to CBX but has no effect on gap junctions, did not alter the effect of acute HS on VP synthesis and release. This reduces the possibility that the inhibitory effect of CBX occurred via a certain non-specific drug effect that is not associated with gap junction blockade (more details in discussion). A scrambled Gap26 peptide also used as a control had a minimal effect on VP synthesis and release induced by acute hyperosmotic stimulus (data not shown).

Taken together, these results indicated that blocking Cx43 hemichannels and gap junctions attenuated acute HS-induced VP upregulation in the SON and plasma.

High extracellular $\mathrm{Ca}^{2+}$ decreases the acute hyperosmotic stimulus-induced VP upregulation in the SON and plasma. Studies have demonstrated that the opening probability of Cx43 hemichannels or gap junctions is markedly reduced when the $\left[\mathrm{Ca}^{2+}\right]_{\mathrm{o}}$ is $>1 \mathrm{mM}$ (14-16). To assess the effect of different $\left[\mathrm{Ca}^{2+}\right]_{0}$ on the acute HS-induced VP upregulation in the SON and plasma, artificial CSF was pre-injected with 0,1 or $2.5 \mathrm{mM}$ $\mathrm{Ca}^{2+}$ into the lateral ventricle of the rats. As expected, there was a marked induction of VP by HS in the SON and plasma of the $\mathrm{HS}+0\left[\mathrm{Ca}^{2+}\right]_{0}$ group (number of VP-positive neurons, $74 \pm 5$, per section; MFI, 313 \pm 28 A.U.; VP concentration in plasma, $12.89 \pm 2.3$ pg/ml; Figs. 1G, 2 and 3). By contrast, artificial CSF with $2.5 \mathrm{mM} \mathrm{Ca}^{2+}$ pretreatment suppressed the induction of VP expression in the SON by $\sim 50 \%$ (35 \pm 7 VP-positive cells, per section; Figs. 1I and 2A), the MFI of positive neurons by

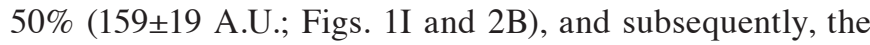
plasma concentration of VP by $50 \%(6.28 \pm 1.13 \mathrm{pg} / \mathrm{ml}$; Fig. 3$)$. Furthermore, the effect of $\mathrm{Ca}^{2+}$ on the HS-induced increase in VP levels was concentration-dependent. Semi-quantitative PCR analysis also revealed similar results to those of the immunostaining and radioimmunoassay. Pretreatment with $2.5 \mathrm{mM} \mathrm{Ca}^{2+}$ reduced acute HS-induced VP synthesis, while 1 or $0 \mathrm{mM} \mathrm{Ca}^{2+}$ had a weak or no effect (Fig. 4).

In conclusion, these results indicated that the opening of Cx43 channels is required for the acute hyperosmotic stimulus-induced VP upregulation in the SON and plasma.

\section{Discussion}

It is well established that the production of vasopressin varies in accordance with changes in the osmotic pressure of the plasma as hypertonic stimuli enhance, whereas hypotonic stimuli decrease the synthesis and release of VP $(1,17)$. The importance of astrocytes in regulating hypotonicity has been well documented $(2,18)$, while the mechanism by which astrocytes regulate hypertonicity remains to be elucidated. Several recent 
studies have demonstrated that the SON astrocytes express c-fos, GFAP and Cx43. Inhibition of astrocyte activity with FCA prevented the activation of MNCs and attenuated the release of VP (7). In addition, blocking $\mathrm{Cx} 43$ interfered with the hyperosmotic stimulus-induced increase in Fos protein expression in MNCs, suggesting that $\mathrm{Cx} 43$ may be involved in the regulation of VP synthesis and release under hyperosmotic stimulus.

In the present study, the role of $\mathrm{Cx} 43$ in the synthesis and release of VP by MNCs following acute hyperosmotic stimulus in vivo was directly examined. $\mathrm{Cx} 43$ is a biogenetic precursor of gap junctions, which are the main connexons expressed in astrocytes (11-13). It has been reported that Cx43 hemichannels are sensitive to various stimuli, including osmotic stimulation (19). A connexin hemichannel spans unopposed plasma membrane regions and, following docking with partners, produces a gap junction channel that allows for direct intercellular communication. In the present study, a Cx43-specific ASODN was utilized to selectively prevent the translation of $\mathrm{Cx} 43 \mathrm{mRNA}$ and reduce the expression of Cx43. It was noted that Cx43-specific ASODN significantly attenuated the HS-induced upregulation of VP levels in MNCs and plasma, indicating that the expression of $\mathrm{Cx} 43$ is necessary for acute HS-induced VP synthesis and release.

Gating of connexin hemichannels in the plasma membrane is modulated by ionic, osmotic, mechanical and oxidative stress, and the prolonged presence of open connexin hemichannels contributes to apoptosis $(20,21)$. The opening of $\mathrm{Cx} 43$ hemichannels has been demonstrated to be induced by hyperosmolarity (22); however, the mechanism by which it occurs remains unknown. Diverse actions are attributed to the opening of $\mathrm{Cx} 43$ hemichannels, including the uptake and release of small molecules, including glutamate and adenosine triphosphate, and the passage of current (12). These actions are inhibited by hemichannel and gap junction blockers. In the present study, CBX and Gap26 were used to block gap junctions and $\mathrm{Cx} 43$ hemichannels, respectively. Pre-treatment with CBX or Gap26 significantly decreased acute HS-induced VP synthesis and release, suggesting that opening of the $\mathrm{Cx} 43$ hemichannel or gap junction is important for the VP synthesis and release induced by acute HS.

$\mathrm{CBX}$ is the most commonly used gap junction and $\mathrm{Cx}$ hemichannel blocker. It is considered that CBX closes gap junctions and $\mathrm{Cx}$ hemichannels by inserting into the plasma membranes (lipid bilayers) and changing the membrane properties in a manner that affects $\mathrm{Cx}$ hemichannel structure and function (23). Previously, this non-specific mechanism of action has been reported to have other effects independent of its blockade of Cx channels, such as being markedly effective at blocking $\mathrm{P} 2 \mathrm{X} 7 \mathrm{R}$ pore formation or volume-regulated anion channels (VRAC) (24). Therefore, it remains a possibility that CBX inhibits the HS-induced VP synthesis and release by blocking P2X7R or VRAC. Since hypertonicity shrinks astrocytes, it is impossible for VRAC to be involved in HS-induced VP synthesis and release (25). Therefore, it is possible to rule out any non-specific effect of CBX on VRAC. In future studies, the next step must therefore be to investigate the role of P2X7R in the HS-induced VP synthesis and release. Cx43/P2X7R-knockout mice may be possible alternative strategies to examine this (26).
Opening of Cx43 hemichannels has been demonstrated during cell volume regulation in response to small changes in $\left[\mathrm{Ca}^{2+}\right]_{\mathrm{o}}$ (between 1.6 and $1.8 \mathrm{mM}$ ) under isosmotic conditions (27). In the present study, it was identified that $2.5 \mathrm{mM}$ $\mathrm{Ca}^{2+}$, a concentration which was expected to reduce channel opening, attenuated the HS-induced VP synthesis and release. A previous study by our group demonstrated that HS induces glutamate release through the $\mathrm{Cx} 43$ hemichannel in cultured astrocytes (28). Therefore, it is likely that glutamate release through the open $\mathrm{Cx} 43$ hemichannel is induced by HS and acts on the MNCs, which synthesize and release VP.

Although astrocytes in the SON may receive osmotic information from the peripheral osmoreceptors and then regulate MNCs (6), MNCs may also receive osmotic information independently (29). This may explain why blockage of the Cx43 hemichannel, reduced opening of the gap junction or inhibition of $\mathrm{Cx} 43$ expression may have, in part but not entirely, inhibited the HS-induced VP level increase in the present study.

Injected hypertonic saline is supposed to act as not only an osmotic stimulus but also as a pain- and stress-inducing agent (30). By contrast to the critical role of plasma osmolality in the regulation of VP release, the effect of stress, such as pain, on VP levels is ambiguous (31). It was demonstrated that injection of isotonic saline had no significant effect on plasma VP levels compared with that of normal rats. Despite this, the possibility that the pain accompanying hypertonic saline injection had an effect on the VP levels cannot be excluded. The experimental design of the present study ensured that the effect of the reagents themselves on VP synthesis and release were excluded.

In conclusion, the present study provided evidence that: i) The expression of Cx43 is necessary for the synthesis and release of VP induced by acute hyperosmotic stimulus; ii) blocking the $\mathrm{Cx} 43$ hemichannel or gap junction limits the acute hyperosmotic stimulation-induced VP synthesis and release by MNCs and iii) opening of the $\mathrm{Cx} 43$ hemichannel or gap junction is required for the acute hyperosmotic stimulus-induced upregulation of VP in SON and plasma.

\section{Acknowledgements}

This study was supported by the National Natural Science Foundation of China (grant nos. 81071595 and 81201514).

\section{References}

1. Bourque CW, Oliet SH and Richard D: Osmoreceptors, osmoreception and osmoregulation. Front Neuroendocrinol 15: 231-274, 1994.

2. Hussy N, Deleuze C, Desarménien MG and Moos FC: Osmotic regulation of neuronal activity: a new role for taurine and glial cells in a hypothalamic neuroendocrine structure. Prog Neurobiol 62: 113-134, 2000.

3. Decavel C and Hatton GI: Taurine immunoreactivity in the rat supraoptic nucleus: prominent localization in glial cells. J Comp Neurol 354: 13-26, 1995.

4. Duan L, Yuan H, Su CJ, et al: Ultrastructure of junction areas between neurons and astrocytes in rat supraoptic nuclei. World J Gastroenterol 10: 117-121, 2004.

5. Yuan H, Duan L, Qiu Y, et al: Response of son astrocytes and neurons to hyperosmotic stimulation after carbenoxolone injection into the lateral ventricle. Acta Anatomica Sinica 35: 127-131, 2004. 
6. Xiong Y, Liu R, Xu Y, et al: Effects of vagotomy, splanchnic nerve lesion, and fluorocitrate on the transmission of acute hyperosmotic stress signals to the supraoptic nucleus. J Neurosci Res 89: 256-266, 2011

7. Yuan H, Gao B, Duan L, et al: Acute hyperosmotic stimulus-induced Fos expression in neurons depends on activation of astrocytes in the supraoptic nucleus of rats. J Neurosci Res 88: 1364-1373, 2010.

8. Qiu C, Coutinho P, Frank S, et al: Targeting connexin43 expression accelerates the rate of wound repair. Curr Biol 13: 1697-1703, 2003

9. No authors listed: Society for Experimental Biology \& Medicine. 1993. Constitution and bylaws. Membership directory. Proc Soc Exp Biol Med 201: D1-D47, 1992.

10. Danesh-MeyerHV,Huang R,Nicholson LF, Green CR: Connexin43 antisense oligodeoxynucleotide treatment down-regulates the inflammatory response in an in vitro interphase organotypic culture model of optic nerve ischaemia. J Clin Neurosci 15 1253-1263, 2008.

11. Bennett MV, Contreras JE, Bukauskas FF and Sáez JC: New roles for astrocytes: gap junction hemichannels have something to communicate. Trends Neurosci 26: 610-617, 2003.

12. Sáez JC, Contreras JE, Bukauskas FF, et al: Gap junction hemichannels in astrocytes of the CNS. Acta Physiol Scand 179: 9-22, 2003.

13. Thimm J, Mechler A, Lin H, et al: Calcium-dependent open/closed conformations and interfacial energy maps of reconstituted hemichannels. J Biol Chem 280: 10646-10654, 2005.

14. Ripps H, Qian H and Zakevicius J: Pharmacological enhancement of hemi-gap-junctional currents in Xenopus oocytes. J Neurosci Methods 121: 81-92, 2002.

15. Stout $\mathrm{C}$ and Charles A: Modulation of intercellular calcium signaling in astrocytes by extracellular calcium and magnesium. Glia 43: 265-273, 2003.

16. Sáez JC, Retamal MA, Basilio D, Bukauskas FF and Bennett MV Connexin-based gap junction hemichannels: gating mechanisms. Biochim Biophys Acta 1711: 215-224, 2005.

17. Zemo DA and McCabe JT: Transcriptional responses of the rat vasopressin gene to acute and repeated acute osmotic stress. Neurosci Res 44: 45-50, 2002.

18. Xiong JJ and Hatton GI: Differential responses of oxytocin and vasopressin neurons to the osmotic and stressful components of hypertonic saline injections: a Fos protein double labeling study. Brain Res 719: 143-153, 1996.

19. Christ GJ, Day NS, Day M, et al: Increased connexin43-mediated intercellular communication in a rat model of bladder overactivity in vivo. Am J Physiol Regul Integr Comp Physiol 284: R1241-R1248, 2003

20. Goodenough DA and Paul DL: Beyond the gap: functions of unpaired connexin channels. Nat Rev Mol Cell Biol 4: 285-294, 2003.
21. Evans WH, De Vuyst E and Leybaert L: The gap junction cellular internet: connexin hemichannels enter the signalling limelight. Biochem J 397: 1-14, 2006.

22. John S, Cesario D and Weiss JN: Gap junctional hemichannels in the heart. Acta Physiol Scand 179: 23-31, 2003.

23. Rozental R, Srinivas M and Spray DC: How to close a gap junction channel. Efficacies and potencies of uncoupling agents. Methods Mol Biol 154: 447-476, 2001.

24. Suadicani SO, Brosnan CF and Scemes E: P2X7 receptors mediate ATP release and amplification of astrocytic intercellular $\mathrm{Ca}^{2+}$ signaling. J Neurosci 26: 1378-1385, 2006.

25. Takano T, Kang J, Jaiswal JK, et al: Receptor-mediated glutamate release from volume sensitive channels in astrocytes. Proc Natl Acad Sci USA 102: 16466-16471, 2005.

26. Giaume $\mathrm{C}$ and Theis $\mathrm{M}$ : Pharmacological and genetic approaches to study connexin-mediated channels in glial cells of the central nervous system. Brain Res Rev 63: 160-176, 2010.

27. Quist AP, Rhee SK, Lin H and Lal R: Physiological role of gap-junctional hemichannels. Extracellular calcium-dependent isosmotic volume regulation. J Cell Biol 148: 1063-1074, 2000.

28. Cao R, Jiang S, Duan L, et al: Hypertonic stimulation induces synthesis and release of glutamate in cultured rat hypothalamic astrocytes and C6 cells. Neurosci Bull 24: 359-366, 2008.

29. Baertschi AJ and Pence RA: Gut-brain signaling of water absorption inhibits vasopressin in rats. Am J Physiol 268: R236-R247, 1995.

30. Larsen PJ and Mikkelsen JD: Functional identification of central afferent projections conveying information of acute 'stress' to the hypothalamic paraventricular nucleus. J Neurosci 15: 2609-2627, 1995

31. Ueta Y, Dayanithi G and Fujihara H: Hypothalamic vasopressin response to stress and various physiological stimuli: visualization in transgenic animal models. Horm Behav 59: 221-226, 2011.

32. Jiang S, Yuan H, Duan L, et al: Glutamate release through connexin 43 by cultured astrocytes in a stimulated hypertonicity model. Brain Res 1392: 8-15, 2011.

33. Lan L, Yuan H, Duan L, et al: Blocking the glial function suppresses subcutaneous formalin-induced nociceptive behavior in the rat. Neurosci Res 57: 112-119, 2007.

34. Frantseva MV, Kokarovtseva L and Perez Velazquez JL: Ischemia-induced brain damage depends on specific gap-junctional coupling. J Cereb Blood Flow Metab 22: 453-462, 2002.

35. Liao $\mathrm{CH}, \mathrm{Wu} \mathrm{Zl}$ and Zhao G: Influence of Connexin 43 on brain edema after acute water intoxication in rats. J Fourth Mil Med Univ 29: 1226-1228, 2008.

36. Wu ZL, Liao CH and Ren N: Study on correlation between connexin43 and brain edema in experimental brain injury. Chin J Neurosurg Dis Res 7: 201-204, 2008. 